\title{
Neurobrucellosis Infection of Ventriculoperitoneal Shunt Presenting as Peritonitis
}

\author{
K. C. Sudhamshu ${ }^{1}$ • A. R. Prasanna Kumar ${ }^{1} \cdot \operatorname{Mary}_{\text {Dias }^{2}}{ }^{2}$ A. M. Shubha ${ }^{1}$ • \\ Kanishka Das ${ }^{1}$
}

Received: 1 December 2015 / Accepted: 21 April 2016/Published online: 4 May 2016

(C) Dr. K C Chaudhuri Foundation 2016

To the Editor: Brucellosis is an endemic zoonosis in developing countries with a worldwide incidence of $<0.01$ to 200 per 100,000 [1]. Brucellosis can involve any organ system and commonly presents with fever and joint pains; central nervous system involvement occurs in $<2 \%[2,3]$.

An 8-y-old boy with congenital hydrocephalus, an indwelling ventriculoperitoneal shunt since infancy and sporadic seizures since 4 y presented with status epilepticus despite antiepileptic therapy. Though the preliminary CSF aspirate from the shunt chamber was reported sterile, there was a growth of Brucella species on further incubation. Three months later, he presented with gross exudative ascites and peritonitis. The peritoneal end of the shunt was diverted for a temporary external drainage and the voluminous ascites was drained with a tube drain. Blood, CSF and ascitic fluid yielded growth of Gram negative coccobacilli which was catalase, oxidase and urease positive. The organism was confirmed as Brucella species by Vitek Agglutination test (1:5120), the test being positive for dilutions exceeding 1:160.

A diagnosis of neurobrucellosis and shunt infection was made and managed with Gentamicin, Rifampicin and Doxycycline and serial CSF monitoring [4]. In the interim, the tube drainage subsided and was removed. He remained asymptomatic; the hydrocephalus was static clinically and radiologically. After $3 \mathrm{wk}$ and sterile cultures (blood, CSF), the shunt assembly was revised and internalized. He received

Kanishka Das

kanishkadas@hotmail.com

1 Department of Pediatric Surgery, St. John's Medical College Hospital, St. John's National Academy of Health Sciences, Bangalore, Karnataka 560034, India

2 Department of Microbiology, St. John's Medical College Hospital, Bangalore, Karnataka, India
Rifampicin and Doxycycline for a total of 6 mo; a repeat CSF culture was negative. At a year follow up, he is asymptomatic and the shunt is clinically normal.

The disease is frequently missed due to lack of suspicion, non-specific features on imaging and stringent laboratory methods. There are six anecdotal reports of Brucella infection with CSF shunts. The earliest report alludes to colonization in a ventriculoatrial shunt in 1981 [5]. Chowdhary et al. reported a 4-mo-old with VP shunt and brucellosis managed with sole medical measures [3]. Later reports by Abdinia et al. [4] and Al- Otaibi et al. [2] stress on shunt replacement in addition to drug therapy. This report documents a ventriculoperitoneal shunt infection with Brucella species presenting as peritonitis and its management with anti-Brucella chemotherapy and temporary CSF drainage.

\section{Compliance with Ethical Standards}

Conflict of Interest None.

Source of Funding None.

\section{References}

1. Mantur BG, Amarnath SK. Brucellosis in India - a review. J Biosci. 2008;33:539-47.

2. Al-Otaibi A, Almuneef M, Shaalan MA. Brucella melitensis infection of ventriculo-peritoneal shunt: a form of neurobrucellosis manifested as gastrointestinal symptoms. J Infect Public Health. 2014;7:62-5.

3. Chowdhary UM, Twum DK. Brucella meningoencephalitis associated with cerebrospinal fluid shunt in a child: case report. Surg Neurol. 1991;35:468-70.

4. Abdinia B, Barzegar M, Malaki M, Behbod H, Oskoui S. Association of Brucella meningoencephalitis with cerebrospinal fluid shunt in a child: a case report. Iran J Child Neurol. 2013;7:35-8.

5. Puri P, Harvey TW. Colonisation of ventriculoatrial shunt with Brucella abortus. Br Med J (Clin Res Ed). 1981;282:1754-5. 